\title{
Laser induced breakdown spectroscopy application in joint European torus 败
}

\author{
A. Semerok ${ }^{\mathrm{a}, *}$, D. L'Hermite ${ }^{\mathrm{b}}$, J.-M. Weulersse ${ }^{\mathrm{a}}$, J.-L. Lacour ${ }^{\mathrm{b}}$, G. Cheymol ${ }^{\mathrm{a}}$, M. Kempenaars ${ }^{\mathrm{c}}$, \\ N. Bekris ${ }^{\mathrm{d}}$, C. Grisolia ${ }^{\mathrm{e}}$ \\ ${ }^{a}$ CEA Saclay, DEN/DPC/SEARS/LISL, P.C. 56, 91191 Gif sur Yvette, Cedex, France \\ b CEA Saclay, DEN/DPC/SEARS/LANIE, P.C. 56, 91191 Gif sur Yvette, Cedex, France \\ c EURATOM/CCFE Fusion Association, Culham Science Centre, Abingdon, OX14 3DB, United Kingdom \\ ${ }^{d}$ EUROfus Program Management Unit PMU, ITER Phys Dept, Culham Sci Ctr, Abingdon OX14 3DB, Oxon, United Kingdom \\ e CEA Cadarache, IRFM/DIR, 13108 St Paul lez Durance, Cedex, France
}

\section{A R T I C L E I N F O}

\section{Article history:}

Received 15 January 2016

Received in revised form 3 August 2016

Accepted 5 August 2016

Available online 8 August 2016

\section{Keywords:}

Tritium retention

Deposited layer

LIBS

JET

ITER

\begin{abstract}
A B S T R A C T
The results on the first successful application of Laser Induced Breakdown Spectroscopy (LIBS) for remote in situ diagnostics of plasma facing components (a deposited layer on a divertor tile) in Joint European Torus (JET) are presented. The studies were performed with an available JET EDGE LIDAR laser system. For in-depth analysis of deposited layers on JET divertor tiles, a number of laser shots were applied onto the same divertor place without laser beam displacement. The spectral lines of D, CII and impurity elements ( $\mathrm{CrI}, \mathrm{BeII}, \ldots)$ were identified in a wide spectral range ( $400-670 \mathrm{~nm}$ ). With the increase in a number of laser shots applied onto the same divertor place, we observed consecutive changes in spectral line intensities of deuterium, carbon, and impurities with the appearance of spectral lines of tungsten substrate (WI). In-depth analysis of deposited layers on JET divertor tiles was made on the basis of the spectral line behaviour in reference to the applied laser shots. The possibility of surface cartography with laser beam displacement on the tile surface was demonstrated as well. Based on the results obtained, we may conclude that LIBS method is applicable for in situ remote analysis of deposited layers of JET plasma facing components.
\end{abstract}

(C) 2016 Elsevier B.V. All rights reserved.

\section{Introduction}

Tritium retention on plasma facing components (PFC), such as divertors, antennas, chamber walls, etc., is seen as a very serious problem for a proper and safe operation of thermonuclear installations [1-3]. Tritium cartography is required to determine tritium overall content for consecutive detritiation. Two main types of tritium deposition and retention in tokamak thermonuclear installations may be described as follows. The first one is hydrogen isotopes trapping on plasma facing walls of a vacuum chamber by its implantation and diffusion on approximately $10 \mathrm{~nm}$ depth, while the second one is hydrogen isotopes co-deposition on PFC of a vacuum chamber with an eroded wall material resulted in formation of deposited layers (DL). It was revealed that in tokamak installations, the hydrogen isotopes are mostly trapped in the deposit of the second type (graphite DL with a thickness up to $100 \mu \mathrm{m}$

\footnotetext{
it Selected Paper from the 8th Euro-Mediterranean Symposium on Laser Induced Breakdown Spectroscopy (EMSLIBS 2015), Linz, Austria, 14-18 September 2015.

* Corresponding author.

E-mail address: alexandre.semerok@cea.fr (A. Semerok).
}

for divertor tiles) [4]. Such thick DL are generally friable (porous) and inhomogeneous with hydrogen atomic content (up to 50\%) and other impurities (lower than $1 \%$ ) resulted from PFC erosion. Friable DL are the source of the dusts which may perturb fusion plasma parameters and reduce thermo-fusion efficiency. Thus, it is very important to search for an adequate method for remote and in situ characterisation and control of DL on PFC.

Laser methods for tokamak surface characterisation were under intensive investigations [5-28]. They were aimed to study in situ diagnostics in tokamaks with a particular interest in realisation and test of LIBS design. Our laboratory LIBS experiments with different DL were also aimed to study how quantitative data from LIBS signal and its evolution may be extracted. The studies were made in CEA, France within the frames of EFDA programs and ITER project [5-11]. Feasibility of LIBS remote measurements was demonstrated with nanosecond Nd-YAG lasers (at 5-10 m distance, under a reduced pressure) with the samples from the European tokamaks (TORE SUPRA, France and TEXTOR, Germany) and with ITER-like samples. The laboratory results have demonstrated possibility to distinguish DL from a graphite or metal substrate, at least on the bench. In addition, based on the comparison of evolution of carbon, hydrogen and impurities spectral lines versus the ablated 
depth, it was possible to get information on both composition and thickness of a DL.

For further development of LIBS and in situ application of this method in a future ITER, it is quite important to obtain a DL in-depth characterisation (composition and elemental in-depth distribution), surface cartography, and to determine analytical spectral lines and their properties (lifetime, intensities) under real conditions of a thermonuclear installation. The content of Be atoms as a major element of a future ITER installation (vacuum chamber walls) is of a special analytical interest.

In this paper, we present the results of our study on LIBS method feasibility for in situ analysis of DL from Joint European Torus (JET). JET is the biggest European thermonuclear installation of TOKAMAK type (2.96 m major radius, $1.25-2.10 \mathrm{~m}$ minor horisontal and vertical radii, $100 \mathrm{~m}^{3}$ plasma volume, in operation since 1984). It is necessary to point out that JET parameters and those of the future ITER are quite similar. JET deposited layer composition and properties may be regarded as very representative to those expected in the ITER (Be and $\mathrm{W}$ content). Plasma fusion experiments were performed with $\mathrm{D} / \mathrm{H} / \mathrm{He}$ plasmas ( $10^{-8}$ Bars high vacuum, $3.45 \mathrm{~T}$ toroidal magnetic field) with Be-atoms (Be-powder) injected in the vacuum chamber for chemical neutralisation of the residual oxygen. LIBS measurements were performed with the JET EDGE LIDAR laser system.

To determine the optimal regime of our specific JET in situ measurements, preliminary laboratory LIBS studies (on laser/target interaction regime, collection optics, optical spectrometer features, ICCD-camera and detection regime, analytical wavelengths and spectral line intensities) were made with the tiles from TEXTOR (Jülich, Germany) and TORE Supra (Cadarache, France) tokamaks and with ITER-like samples [5-11]. The laboratory LIBS studies were made with the installation developed earlier in CEA for the Mars surface analysis (the NASA Mars Rover Curiosity mission, within the ChemCam project [29-31]). The developed installation was applicable for our laboratory studies as specific conditions for LIBS application in JET and those for the Mars surface analysis are quite similar (remote measurements, reduced pressure, unknown sample properties).

This paper presents the results on the first successful application of in situ LIBS analyses in JET. The results may be considered as very promising for in situ remote diagnostics of PFC in JET. This is especially true for the cases when a laser beam is targeted at the inner leg of the divertor where heavy deposition is observed. The EDGE LIDAR laser and its collection optics may be used for LIBS measurements. Though any application of new diagnostics on JET seems to be a tough challenging task, we have the reasons to believe that LIBS analyses performed with the EDGE LIDAR laser system may find their application for in situ remote diagnostics of PFC in JET. LIBS-method may also be applicable for other thermonuclear installations (WEST, ITER).

\section{Experiments}

Remote LIBS measurements were performed in JET when plasma and magnetic field were shut off. In the JET design of a vacuum chamber, the optical window (125 mm diameter) had limited apertures. The collection distance from the target (divertor tile) to the window was $5.36 \mathrm{~m}$. LIBS spectrometer was at approximately $7 \mathrm{~m}$ from the optical window. Thus, the total distance of remote measurements was $>10 \mathrm{~m}$. High vacuum environment $\left(10^{-8}\right.$ Bar) in the zone of the analysis and a long collection distance from the laser plasma to collection optics were the particular features of these LIBS measurements in JET.

LIBS analyses were performed on a graphite divertor tile with a Wstripe (20 mm $\times 142 \mathrm{~mm}$ ). The deposit on the $\mathrm{W}$-stripe was formed after a certain number of JET fusion plasma shots with $\mathrm{D} / \mathrm{H} / \mathrm{He}$ mixture. The main deposit components were carbon sputtered from the graphite tiles and hydrogen isotopes used for tokamak fusion plasma (94\% deuterium, $5 \%$ hydrogen, and $1 \% \mathrm{He}$ ). The possible impurity content in the deposits was supposed as $\mathrm{Fe}, \mathrm{Cr}$, Be and other elements associated with the erosion of the PFC materials of a known composition. However, neither the layer thickness, nor the elemental in-depth and surface distributions were known. Thus, the sample under study may be described as a W-stripe with $\mathrm{D} / \mathrm{H} / \mathrm{He}$ and $\mathrm{C}$ deposit (with Be and other elements as impurities) of unknown thickness. Generally, the DL thickness is about $10-100 \mu \mathrm{m}$.

LIBS studies were performed with JET EDGE LIDAR laser system designed for plasma temperature measurements by the Thomson scattering. It comprised a Ruby laser with the following parameters: $3 \pm 0.5 \mathrm{~J}$ per pulse energy, $690 \mathrm{~nm}$ wavelength, 300 ps pulse duration, a near Gaussian beam, a very low repetition rate of 1 shot per 5 min [32]. Laser beam was focused inside the vacuum chamber and reached the divertor tile with the spot of $15-20 \mathrm{~mm}$ diameter. In such a case, the laser fluence on the tile was about $1 \mathrm{~J} / \mathrm{cm}^{2}\left(3 \mathrm{GW} / \mathrm{cm}^{2}\right.$ laser beam intensity), which was not sufficient to create a bright LIBS plasma and to observe any spectral lines emission. To increase the laser beam intensity for our LIBS experiments, the optical scheme of the EDGE LIDAR system was slightly modified without upsetting the operation of the whole system. An additional divergent lens ( $20 \mathrm{~m}$ focal length) was used to fix the laser beam focal point on the divertor tile surface with the spot of $\mathrm{d}=$ $4 \mathrm{~mm}$ diameter, thus allowing us to increase the laser intensity up to $80 \mathrm{GW} / \mathrm{cm}^{2}$, which was sufficient to create the bright laser plasma and to ablate the DL by consecutive pulses. The lens parameters and its position were determined by exhaustive calculations. He-Ne alignment laser (coaxial to the Ruby one) was visible on a control camera used to monitor laser beam displacement on the tiles surface.

\subsection{Spectrometer choice}

As hydrogen isotope content comprised $94 \%$ of deuterium (D) and only $5 \%$ of hydrogen $(\mathrm{H})$, the hydrogen isotope spectral line resolution was of minor importance in our study. To optimize the signal/noise ratio and to distinguish LIBS spectral lines, a spectrometer with high collection efficiency and moderate spectral resolution $(\geq 2000: 1)$ is required for remote measurements without isotope resolution. With this consideration in mind, we have chosen a spectrometer ANDOR Shamrock SR-303i with a large numerical aperture f/4 (as an advantage in our case) and $303 \mathrm{~mm}$ focal length, but with a narrow working spectral range of $22 \mathrm{~nm}$ (as an inevitable disadvantage). The spectral resolution could be varied very easily by setting one of three available gratings ( $300 \mathrm{l} / \mathrm{mm}, 1800 \mathrm{l} / \mathrm{mm}$, and $2400 \mathrm{l} / \mathrm{mm}$ ) and by adjustment of the spectrometer entrance slit width $(50-500 \mu \mathrm{m})$. We applied a $1800 \mathrm{l} / \mathrm{mm}$ grating blazed at $520 \mathrm{~nm}$. A $200 \mu \mathrm{m}$ slit width was chosen for reliable detection of LIBS signals (spectral lines) in the visible spectral range with $\approx 0.3 \mathrm{~nm}$ spectral resolution. It should be noted that for the spectrometer used in our studies, we have observed a certain asymmetry of the spectral lines in 630-660 nm spectral range which resulted in the deformation of the spectral shape of $\mathrm{D}_{\alpha}$ line $(656 \mathrm{~nm})$. Taking into account that the spectrometer was rented solely for the JET experiments, the particular feature of this asymmetry and the reason of its appearance was disregarded.

\subsection{Optical detector choice}

To decide between EMCCD and ICCD camera available for ANDOR Shamrock spectrometers, our considerations were as follows. EMCCD for detection of a low flux of photons has an advantage of a slightly better signal/noise ratio, but the difference is less than a factor of two. With ICCD camera ( $13.5 \mu \mathrm{m}$ pixel size), the loss of spectral resolution was not important for our experiments. In addition, with ICCD camera, we could use the time gating which provides the possibility of temporal resolution of the spectrum and a direct (without optical filters) spectral analysis near the laser beam wavelength. Photon counting may be used for a very low signal. Thus, ICCD camera was preferred for our experiments. The camera comprised an intensifier with multi-channel plates (MCP) of $18 \mathrm{~mm}$ diameter. The intensifier was trigged by a delay generator which itself was triggered by an electric signal from the nearby Ruby 
laser. The triggering signal from laser arrived $50 \mathrm{~ns}$ prior to the light from the LIBS plasma.

The ICCD may be calibrated at a low photon flux. From the spectrum recorded with a short time gate, we can count the electrons emitted by the photo cathode and measure the signal in terms of counts $\times$ pixels. Each electron generates about 2 counts $\times$ pixels with MCP gain of 25 and 23 counts $\times$ pixels with MCP gain of 100 .

\subsection{Collection system}

One of six lenses (125 mm diameter) of the available EDGE LIDAR collecting system was used for light collection. With LIBS optical scheme, it was possible to image the laser plasma plume at the entrance of ANDOR Shamrock SR-303i spectrometer with the total $1 / 12 \times$ magnification. We were able to image it with the spectrometer ICCD camera by using the grating at a zero order. The plasma plume size on the entrance of the LIBS spectrometer was of $1.06 \mathrm{~mm}$ (FWHM). Thus, the estimated plasma plume diameter $(\varnothing)$ was about $12.7 \mathrm{~mm}$. The numerical aperture of the collection system was $1 / 43$ (125 mm diameter lens at $5.36 \mathrm{~m})$. With the total $1 / 12 \times$ magnification, the resulting numerical aperture of the collection optics was $1 / 3.6$ which fitted quite well a 1 / 4 numerical aperture of the spectrometer for efficient light collection.

\subsection{Alignment procedure}

The optical scheme for collection optics alignment is presented in Fig. 1. With a sliding mirror (M5) positioned at the spectrometer entrance, it was possible to provide the alignment by auto collimation of the second He-Ne laser (installed on the bench). The laser beam was set parallel to the spectrometer axis to allow its pass in the centre of the slit and then near the centre of lenses and mirrors. A mirror (M1) was then positioned and oriented in such a way that the alignment He-Ne beam was allowed to follow the EDGE LIDAR optical collection path. The spot of the He-Ne beam on the tile could be easily observed on the monitor of the JET camera. Then the alignment procedure was continued to move and rotate a mirror (M2) to superpose this spot on the one of the alignment beam of the EDGE LIDAR laser. Some rotations of both mirrors (M1 and M2) were necessary. The above procedure with 200-500 $\mu \mathrm{m}$ spectrometer slit width has always resulted in a good LIBS signal. The position of the plasma image at the spectrometer entrance was controlled and checked with the spectrometer in a zero order of grating. While moving the EDGE LIDAR laser spot on the tile (for surface cartography), the EDGE LIDAR collection system was realigned by moving the He-Ne spot on the one of EDGE LIDAR alignment lasers. An opaque protective material to cover the measuring devices was used during all manipulation procedures of our experiments.

\section{Experimental results}

The detection regime was adjusted by varying the spectrometer and ICCD camera in their parameters ranges: spectrometer slit width (50$500 \mu \mathrm{m})$, ICCD camera delay (180-680 ns), ICCD camera gate width (2-10 $\mu \mathrm{s})$ and MCP gain (25-250). With the adjusted detection parameters (200 $\mu \mathrm{m}$ spectrometer slit width, 180 ns ICCD camera delay, and 25 or 100 MCP gain), we observed the spectra with well resolved spectral lines of a high intensity and without saturation. The ICCD camera gate width did not affect the spectra. It was chosen as $10 \mu$ s to make sure that all photons from the spectral lines with different lifetimes have been detected. Registration tests were made with a laser pulse energy $\mathrm{E}=3.2 \pm 0.5 \mathrm{~J}$ following each laser shot $\left(\mathrm{n}^{\text {th }}\right)$ in a set of consecutive laser shots (up to ten on the selected zone). The registration was made on the divertor surface with a DL on the W-stripe. Fig. 2 presents the examples of LIBS spectra with $D_{\alpha}, D_{\beta}$ and $D_{\gamma}$ deuterium atoms spectral lines registered after optimisation of the ICCD camera and spectrometer parameters.

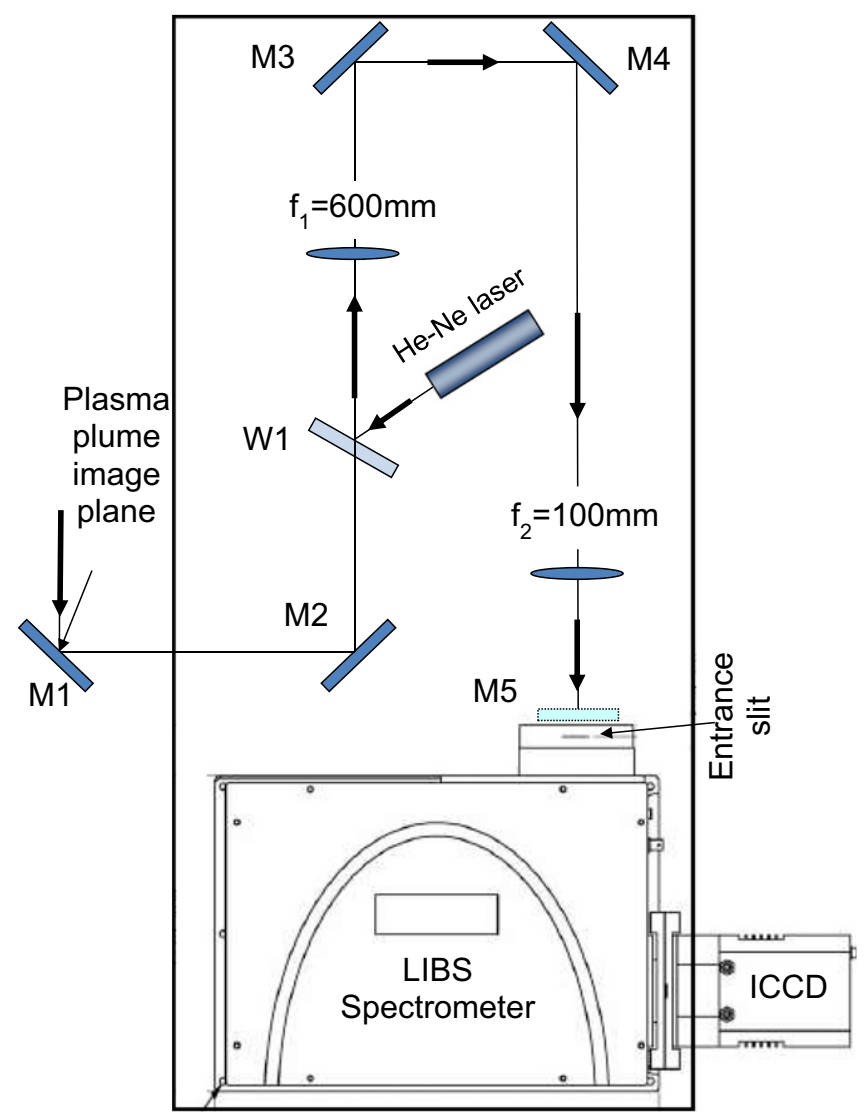

Fig. 1. Optical scheme for collection optics alignment. M1-M4 - reflecting mirrors, M5 sliding mirror, $\mathrm{W} 1$ - semi-transparent mirror, $\mathrm{f}_{1}$ and $\mathrm{f}_{2}$ - lenses.

LIBS in-depth characterisation of a virgin DL on the W-stripe was made in two neighbouring zones (zone 1 and 2, being $10 \mathrm{~mm}$ distance apart).

For zone 1, ten laser shots were made onto the same divertor surface without laser beam displacement. Each spectrum was registered following each laser shot. For in-depth characterisation, the spectral range of $425-440 \mathrm{~nm}$ with the spectral lines of $\mathrm{D}_{\gamma}, \mathrm{CII}, \mathrm{CrI}$, and BeII was used (Fig. 2c). With the increase in the number of laser shots without the laser displacement, the deuterium line intensity was decreasing with the appearance of tungsten (WI) spectral lines with the 5th-6th laser shot (Figs. 3 and 4). The spectral line intensities of the impurities $(\mathrm{Cr}$, Be) were decreasing somewhat slower.

For LIBS in-depth characterisation of a virgin DL on the W-stripe on zone 2 (at $\approx 10 \mathrm{~mm}$ distance from zone 1 ), a set of only four consecutive laser shots was made. This was associated with the necessity to respect rigid time limitations authorized for our experiments. The spectrum registration procedure was the same as for zone 1 - each spectrum was registered following each laser shot onto the same divertor surface (without laser beam displacement). The spectral range of 650-660 nm with the spectral lines of $\mathrm{D}_{\alpha}$ and CII was used (Fig. 2a). LIBS spectra of deuterium and carbon after the 1st - 4th laser shot are presented on Fig. 5 (a-d, respectively). Saturated $D_{\alpha}$ spectral line from the 1 st laser shot (Fig. 5a) was approximated by fitting the typical spectral broadening profiles of $\mathrm{D}_{\alpha}$ lines of non-saturated signals. The obtained deuterium and carbon in-depth distributions are presented in Fig. 6.

In LIBS application for tritium inventory, the determination of lifetime of hydrogen isotope spectral line was of high interest. The lifetime of deuterium $\mathrm{D}_{\alpha}$ spectral line ( $656 \mathrm{~nm}$ ) was determined experimentally by detection of the line intensity at different ICCD camera delays (180 ns, 380 ns, 780 ns, and 2180 ns) with $10 \mu$ sate width. Spectral line behaviour with the time delay was obtained by subtracting consecutive $D_{\alpha}$ spectra ( $D_{\alpha}$ spectral line amplitudes in our case). The obtained 

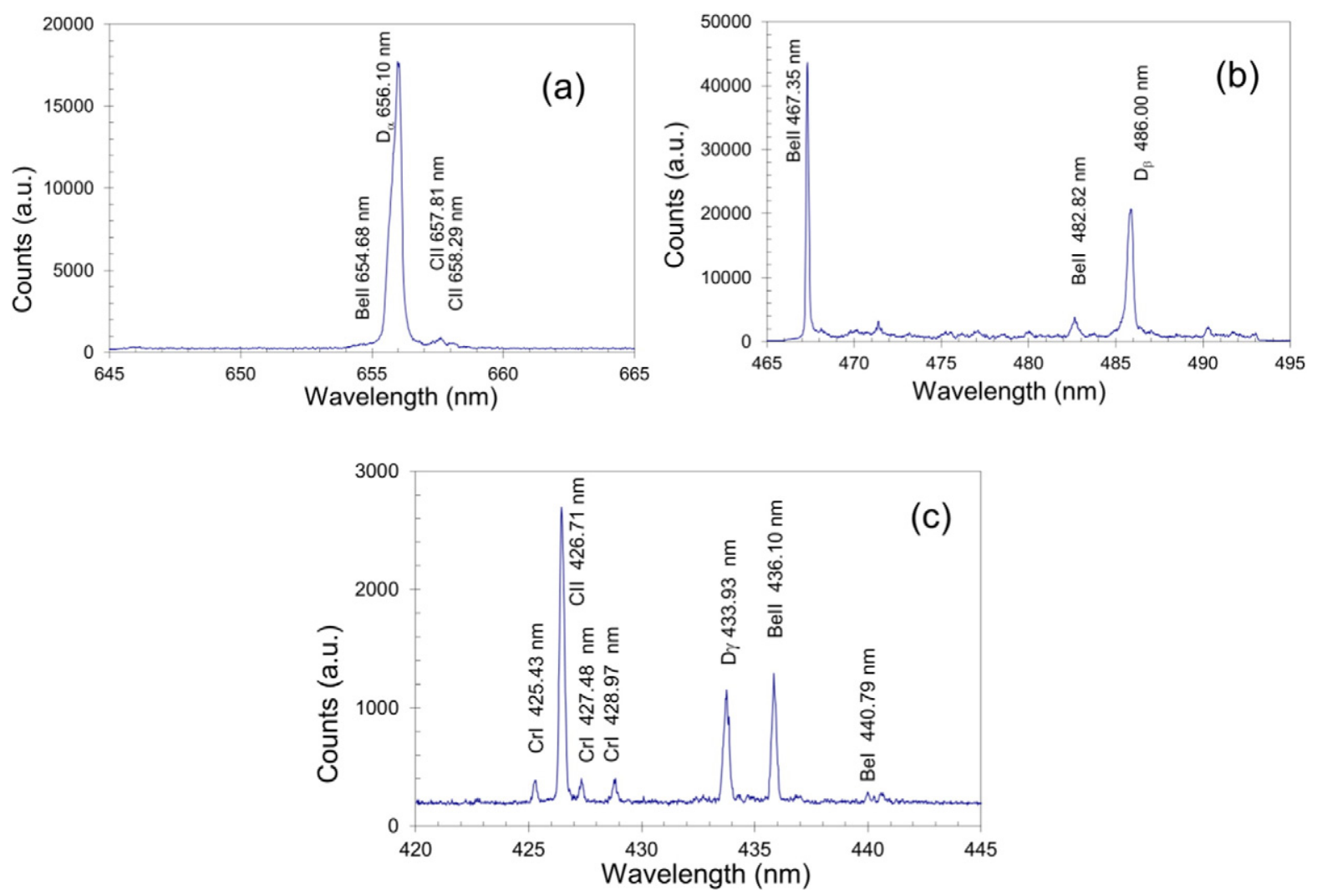

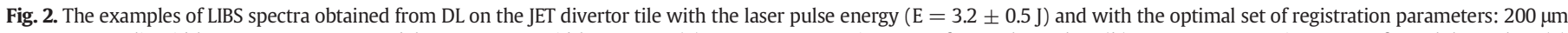

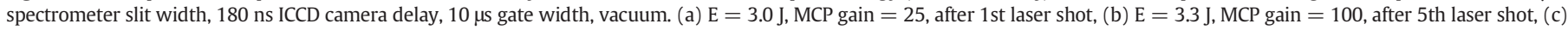
$\mathrm{E}=3.0 \mathrm{~J}$, MCP gain $=100$, after 1 st laser shot.

$\mathrm{D}_{\alpha}$ spectral line evolution was decreasing with the time delay (Fig. 7, solid line rectangulars). From this spectral line behaviour, it was possible to derive $\mathrm{D}_{\alpha}$ lifetime as $\tau=700 \pm 100 \mathrm{~ns}$.

Table 1 presents LIBS spectral lines obtained for 360-665 nm spectral range. Solely intensive and well distinguished spectral lines are presented. Spectral line reference data are taken from [33].

\section{Discussion}

Our investigations were aimed to study feasibility of in situ LIBS application in JET. Significant efforts were made to optimize the design and measuring features of the available JET EDGE LIDAR detection

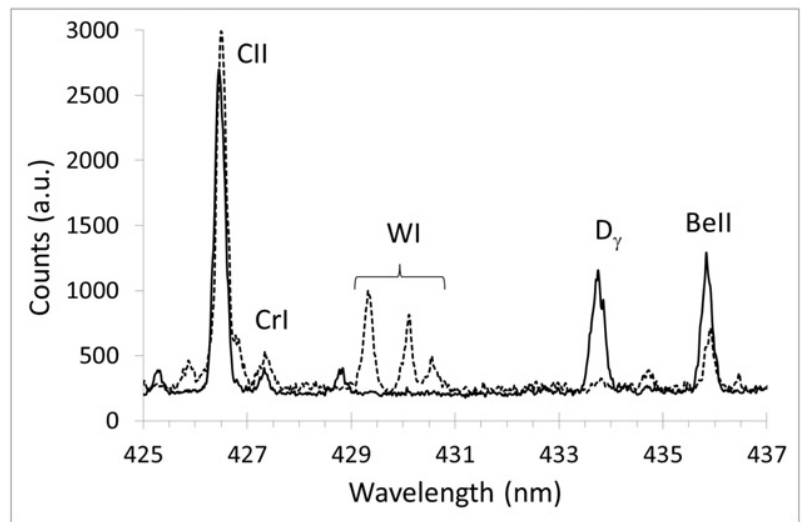

Fig. 3. LIBS spectrum after the 1st (solid curve) and 10th laser shot (pointed curve) on the W-stripe zone with the DL in zone 1 (laser pulse energy $E=3.2 \pm 0.5 \mathrm{~J}, 200 \mu \mathrm{m}$ spectrometer slit width, $1800 \mathrm{~mm}^{-1}$ grating, $180 \mathrm{~ns}$ ICCD camera delay, $10 \mu$ s gate width, $\mathrm{MCP}$ gain $=25$, vacuum). system to characterize a DL on JET divertor by LIBS analytical method. For our LIBS measurements in JET, it was necessary to satisfy rigid limitations imposed by the JET authorities. They were as follows: a limited 5-day period was allowed for LIBS experiments (including installation, adjustment, and measurements); LIBS optical scheme insertion should not upset the operation of the EDGE LIDAR diagnostics scheme; the target (the zone under analysis) was in vacuum chamber at $5 \mathrm{~m}$ distance from the vacuum chamber optical window and without an access for operator. In addition to these limitations and difficulties, we should also mention that in the available EDGE LIDAR system, the laser beam is focalized not on the divertor tiles, but rather on the fusion plasma. This particular feature of the system results in the limited intensity on the target (divertor tile) which was not enough for LIBS analyses. In our previous experiments in JET, it was revealed that a large laser spot (15-20 mm diameter) does not create any detectable LIBS signal. To

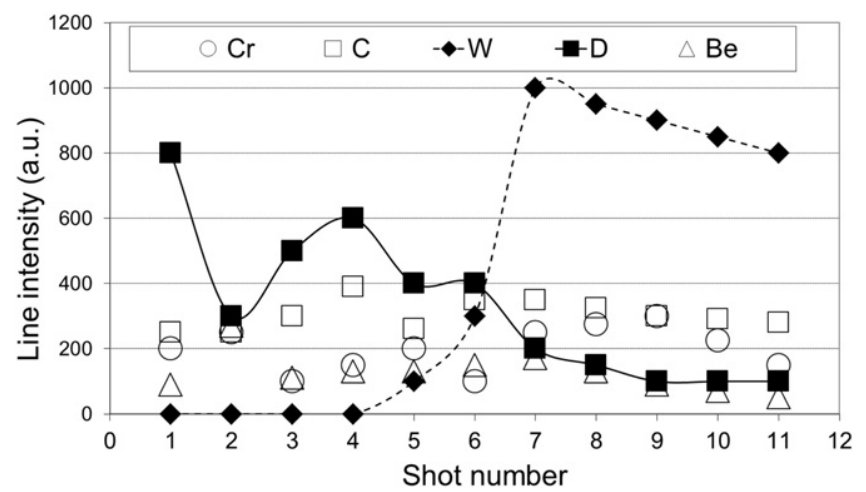

Fig. 4. In-depth characterisation of the DL (zone 1). $\mathrm{D}_{\gamma}$, WI, BeII, CII and CrI spectral line intensities are presented as a function of a number of applied laser shots. 

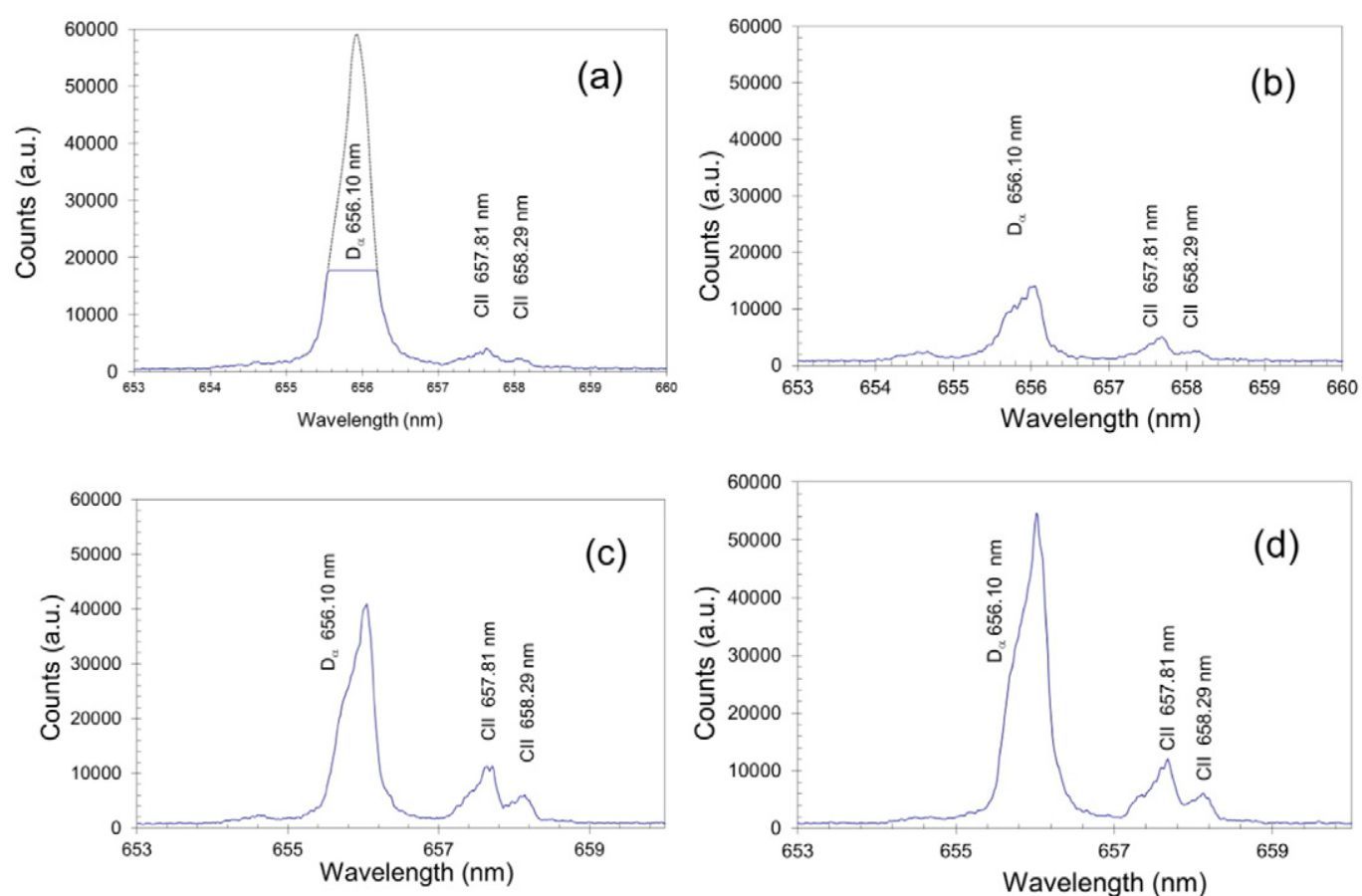

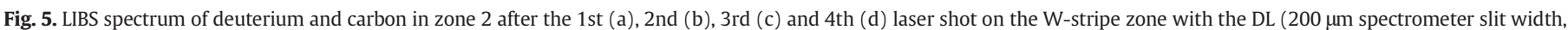

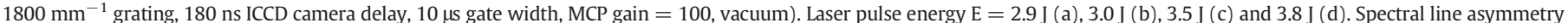
is related with the spectrometer particular feature on the spectral range of 630-660 nm.

increase the laser beam intensity and to make it sufficient for our LIBS measurements, an additional corrective lens with pre-calculated parameters was installed without upsetting the optical scheme of EDGE LIDAR system.

The analysis of deuterium spectral lines (Fig. 2) has revealed that the Stark line broadening was comparable with the spectrometer resolution ( $0.45 \mathrm{~nm}$ and $0.30 \mathrm{~nm}$ for $656 \mathrm{~nm}$ and $434 \mathrm{~nm}$ deuterium wavelengths, respectively). For the spectral transitions of other elements ( $C, \mathrm{~W}, \mathrm{Be})$, we observed that their spectral line broadening was lower than the spectrometer resolution and $\mathrm{D}_{\alpha}$ broadening. Table 1 presents wavelengths and intensities for the well resolved spectral lines registered in our study. This data may be of interest for future LIBS application for JET and ITER deposited layer characterisation and for tritium in situ inventory.

From Fig. 4, one can see that the spectra registered on the same zone following shot-to-shot laser ablation have revealed a non-homogeneous deuterium in-depth distribution in the DL. The obtained indepth distribution of such elements as $\mathrm{H}, \mathrm{C}$ (the major elements) and impurities is not very pronounced and not sharp. This result is also

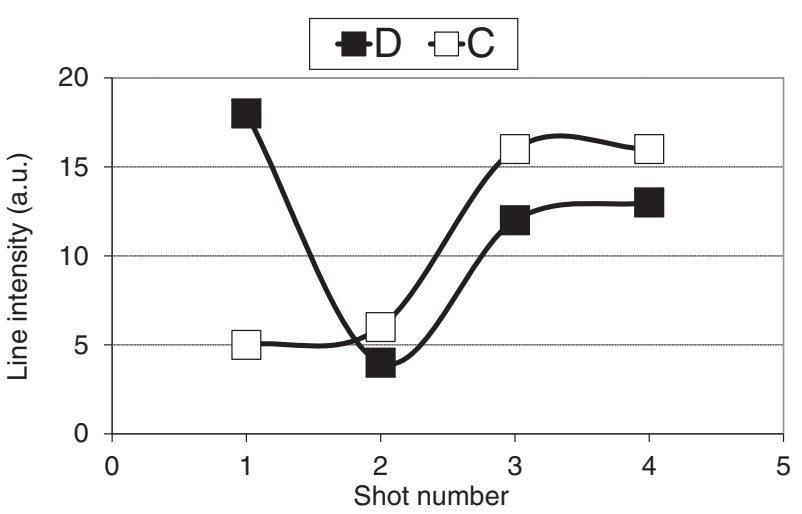

Fig. 6. In-depth characterisation of the DL for zone 2. $D_{\alpha}$ and CII spectral line intensities are presented as a function of a number of applied laser shots. valid for tungsten (that is, the layer substrate). This can be associated with the Gaussian laser beam intensity distribution and with the consecutive non homogeneous in-depth ablation which is more rapid for the central zone with higher laser intensity [8]. One should note that transportation of a top-hat laser beam for remote measurements (10 m distance, in our case) is not possible. To improve in-depth resolution and to provide a more homogeneous laser in-depth ablation with reduced thermal perturbation of hydrogen distribution, LIBS-analysis with ultrashort double pulses may be advised [34-36].

From Figs. 4 and 6, one can see that the spectra registered on the neighbouring zones 1 and 2 ( $10 \mathrm{~mm}$ apart) on the DL have revealed a quite similar in-depth distribution of (D) and (C) for both the zones. Thus, the surface distribution of the deposit may be supposed as sufficiently homogeneous (at least, on the $10 \mathrm{~mm}$ scale).

The results obtained on LIBS-application for DL characterisation on the neighbouring zones may be regarded as a good demonstration of the possibility to make not solely in-depth analysis, but also rapid surface cartography measurements. Rapid cartography should be regarded as obligatory for LIBS-diagnostics for JET and ITER safe operation. Thus,

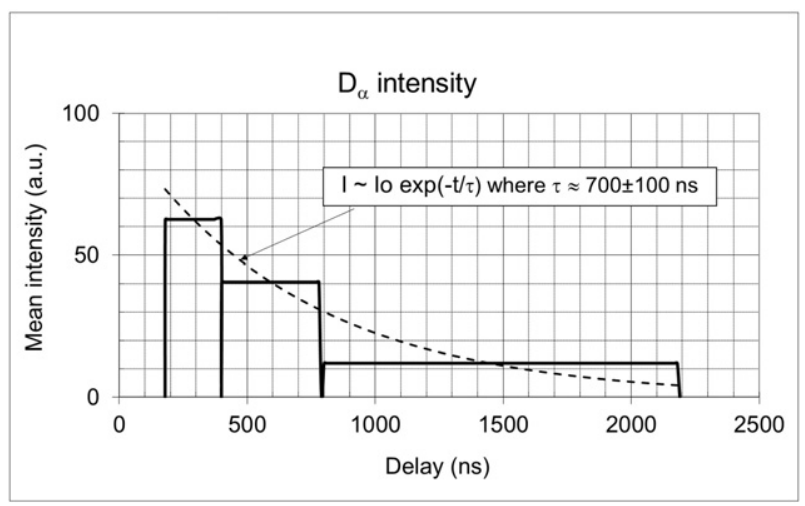

Fig. 7. $D_{\alpha}$ spectral line $(656 \mathrm{~nm})$ decrease with the time delay (solid line rectangulars). An approximation of $D_{\alpha}$ spectral line exponential decrease for the time delay derives $\tau \cong 700 \mathrm{~ns}$ (a dashed line). 
Table 1

Identified LIBS spectral lines detected for divertor DL in JET environmental conditions. Observed spectral line intensities (second column) are related to 25 MCP gain.

\begin{tabular}{|c|c|c|c|c|c|c|}
\hline Wavelength (nm) & Intensity (a.u.) & Transition type & $\mathrm{A}_{\mathrm{ij}}\left(10^{7} \mathrm{~Hz}\right)$ & $E_{j}(e V)$ low & $E_{i}(e V)$ upper & Shot no \\
\hline 658.29 & 400 & C II & 3.66 & 14.45 & 16.331 & 1 \\
\hline 657.81 & 1000 & C II & 3.67 & 14.45 & 16.333 & 1 \\
\hline \multirow{3}{*}{656.10 (multiple) } & 6000 & & & & & 1 \\
\hline & 1400 & $\mathrm{D}_{\alpha}$ & 23 & 10.2 & 12.1 & 2 \\
\hline & 1600 & & & & & 5 \\
\hline 654.8 (multiple) & 80 & Be II & - & 14.81 & 16.7 & 2 \\
\hline 589.59 & 900 & $\mathrm{Na} I$ & 6.14 & 0.0 & 2.10 & 10 \\
\hline 532.83 & 5 & $\mathrm{Cr} \mathrm{I}$ & 6.2 & 2.91 & 5.24 & 3 \\
\hline 529.74 & 10 & $\mathrm{Cr} \mathrm{I}$ & 3.88 & 2.9 & 5.24 & 3 \\
\hline 527 (multiple) & 400 & Be II & 9.7 & 11.96 & 14.32 & 3 \\
\hline 521.82 & 160 & $\mathrm{Cu} \mathrm{I}$ & 7.5 & 3.82 & 6.19 & 3 \\
\hline 515.32 & 100 & $\mathrm{Cu} \mathrm{I}$ & 6.0 & 3.79 & 6.19 & 3 \\
\hline 490-515 (multiple) & 800 & $\mathrm{Ni} \mathrm{I}$ & - & - & $\approx 6$ & 4 \\
\hline 486.00 (multiple) & 1600 & $\mathrm{D}_{\beta}$ & 7.16 & 10.20 & 12.75 & 5 \\
\hline 482.8 (multiple) & 200 & Be II & 1.75 & 12.16 & 14.72 & 5 \\
\hline 467.34 (multiple) & 5000 & Be II & 44.27 & 12.16 & 14.81 & 6 \\
\hline 457.27 & 4500 & Be I & 7.62 & 5.28 & 7.99 & 6 \\
\hline 440.79 & 100 & Be I & 1.01 & 5.28 & 8.09 & 7 \\
\hline 436.1 (multiple) & 2500 & Be II & 21.81 & 11.96 & 14.81 & 2 \\
\hline 433.93 (multiple) & 1000 & $\mathrm{D}_{\gamma}$ & 3.26 & 10.20 & 13.06 & 1 \\
\hline 428.97 & 150 & $\mathrm{Cr} \mathrm{I}$ & 3.16 & 0.0 & 2.9 & 2 \\
\hline 427.48 & 150 & $\mathrm{Cr} \mathrm{I}$ & 3.07 & 0.0 & 2.9 & 2 \\
\hline 426.73 (multiple) & 2000 & C II & 47.69 & 18.05 & 20.95 & 1 \\
\hline 425.43 & 150 & $\mathrm{Cr} \mathrm{I}$ & 3.15 & 0.0 & 2.91 & 2 \\
\hline
\end{tabular}

an optical design without necessity of additional adjustment of optical collection of a laser beam following its displacement on the tested surface is required.

We should stress that LIBS-method feasibility has been demonstrated for solely qualitative characterisation of the layer under extremely severe environmental conditions of JET experiments. Neither a DL thickness, nor its elemental content may be deduced from the obtained results. For DL thickness measurements, a laser ablation rate should be determined. Numerous intensive studies on tokamak DL ablation and reference database creation are required to obtain this unknown feature. For in situ and online (real time) measurements of laser ablation rate, the integration of LIBS-method and speckle interferometry may be advised [37]. For LIBS quantitative measurements of deposited elemental content, two solutions may be advised. The first one is ablation of etalon samples with the known elemental content which is followed by calibration curve method. LIBS studies with ITER-like samples have already been initiated [8]. Calibration-free method may be advised as another solution [11,3839]. In this method, one should consider not solely the existence of a local thermodynamic equilibrium, but also plasma stoichiometry and a possible modification of hydrogen distribution resulting from crater heating by previous laser pulses.

The possibility to make LIBS-measurements with a single laser pulse may be regarded as one of the important results of our study. These measurements were performed following a very thorough parameter optimisation of laser and detection systems. A powerful (3 J) and short pulse (300 ps) laser allowed us to create a bright powerful plasma plume $(\varnothing \approx 10 \mathrm{~mm}$ ) with a controllable ablation rate. The ablation rate may be estimated from a typical layer thickness $(\delta \approx 15 \mu \mathrm{m})$ in the tested zone [40-41]. From Fig. 4, one can see that 5 pulses were required to ablate the DL and to reach the substrate (tungsten). Thus, the laser ablation rate may be estimated as $3 \mu \mathrm{m}$ per pulse. For applied laser beam with $\mathrm{d}=4 \mathrm{~mm}$ spot diameter, ablation rate is $\approx 4 \times 10^{-5} \mathrm{~cm}^{3}$ per pulse, that is, for typical solid matter density of $10^{23}$ atoms $/ \mathrm{cm}^{3}$, we can estimate that $\mathrm{N}_{\text {pulse }} \approx 5 \cdot 10^{18}$ atoms per pulse were ablated. Supposing that total atomisation of this ablated matter took place, one may estimate that plasma plume density was about $\mathrm{N}_{\text {pulse }} /$ $\varnothing^{3} \sim 10^{18}$ atoms $/ \mathrm{cm}^{3}$. Even with $10 \%$ of plasma ionisation for ps laser pulse of $80 \mathrm{GW} / \mathrm{cm}^{2}$ intensity, the electron density was $\sim 10^{17} \mathrm{~cm}^{-3}$, that is, it was sufficient to reach local thermal equilibrium for hydrogen atoms [42].
Plasma plume temperature may be estimated from hydrogen $D_{\alpha}$ spectral line lifetime $(\tau \cong 700 \mathrm{~ns})$ and its diameter $(\varnothing \cong 12.7 \mathrm{~mm})$. Thus, plasma plume expansion velocity was $\mathrm{v}_{\mathrm{p}} \approx \varnothing / \tau \cong 18 \cdot 10^{3} \mathrm{~m} / \mathrm{s}$ and the corresponding deuterium atoms kinetic energy $\mathrm{E}_{\mathrm{k}} \cong 3.4 \mathrm{eV}$. For a laser plasma plume expansion in vacuum, its temperature was of the same order as for kinetic energy $\mathrm{E}_{\mathrm{k}}$ [43-47]. Thus, a mean temperature of LIBS plasma may be estimated as $\mathrm{T} \approx 3 \mathrm{eV}$, though this estimation data should be very carefully verified in future experiments. Such high LIBS plasma temperature is in accordance with the pronounced presence of intense ionic spectral lines (CII and BeII) and those of deuterium with high excitation energy $(\approx 10 \mathrm{eV})$.

The hydrogen isotopes content measurements were of a minor importance in our experiments as the major hydrogen isotopes in the deposits was deuterium (94\%). For LIBS-analysis with the spectral line resolution of $\mathrm{D} / \mathrm{T}$ hydrogen isotope, the spectral resolution of the spectrometer should be better than 10,000:1, and hydrogen spectral line broadening should be less than their isotopes shifts ( $\cong 0.06 \mathrm{~nm}$ for $D_{\alpha} /$ $\mathrm{T}_{\alpha}$ and $\cong 0.04 \mathrm{~nm}$ for $\mathrm{D}_{\beta} / \mathrm{T}_{\beta}$ or $\mathrm{D}_{\gamma} / \mathrm{T}_{\gamma}$ ) [48-49].This resolution may be obtained even with a conventional spectrometer (like the one applied in our studies, but with the slit width of $20 \mu \mathrm{m}$ ). The spectral line broadening in the LIBS plasma may be seen as a major problem for measurements of isotopic resolution. In this case, a more intense hydrogen spectral line $\left(\mathrm{H}_{\alpha}, \mathrm{D}_{\alpha}\right.$, and $\left.\mathrm{T}_{\alpha}\right)$ may not be regarded as the best choice if its Stark broadening is higher than the one for $\mathrm{H}_{\gamma}, \mathrm{D}_{\gamma}$, and $\mathrm{T}_{\gamma}$ transitions (Figs. 2a and 5). Further investigations are required to study the possibility to make measurements of hydrogen isotope content with $\mathrm{D}_{\gamma}$ and $\mathrm{T}_{\gamma}$ (or with $\mathrm{D}_{\beta}$ and $\mathrm{T}_{\beta}$ ) transitions with a less pronounced line broadening (Figs. 2b and c).

Some features of plasma spectroscopy and LIBS-method application in relation to magnetic field effects should be noted. In this case, the magnetic field of some Tesla may result in additional spectral line broadening by the Zeeman splitting. For $\mathrm{D}_{\alpha}$ transitions, this splitting is ( $\approx 0.04 \mathrm{~nm} /$ Tesla) [50] and is comparable with the isotopes shift. For $\mathrm{D}_{\beta}$ and $\mathrm{D}_{\gamma}$ transitions, the Zeeman splitting is lower, but not negligible [51-52]. The Zeeman broadening together with the Doppler and the Stark ones may reduce the spectral line resolution, and, consequently, result in a lower LIBS potentiality for isotopic measurements. This is regarded as a negative effect of the magnetic field. However, with the magnetic field parallel to the sample surface, the LIBS plasma may be confined, thus resulting in a longer lifetime of spectral lines and in 
higher LIBS analytical signals [53-56]. In case of a high magnetic field, one should mention the effect of the Larmor rotational movements of ions as another plasma spectroscopy particularity [57-58]. However, this effect may be neglected in transient (plasma lifetime of $700 \mathrm{~ns}$ ) and collisional LIBS plasma.

To summarise the above considerations, we may conclude that our results on LIBS in situ application in JET may be regarded as very satisfactory and inspiring to continue the multi-aspect study of LIBS analytical method in search for the best efficient design for LIBS diagnostics for tritium inventory in Tokamaks.

\section{Conclusions}

Feasibility of LIBS remote measurements was demonstrated with JET EDGE LIDAR laser system (300 ps Ruby laser, laser intensity up to $80 \mathrm{GW} / \mathrm{cm}^{2}, 10 \mathrm{~m}$ distance, vacuum) and a thick deposited layer $(\sim 10 \mu \mathrm{m})$ on JET divertor tile. A number of analytical spectral lines (D, CII, CrI, and BeII) in 400-600 nm spectral range were distinguished and identified.

Based on the successful qualitative results on in situ LIBS application in JET, we may conclude that LIBS method along with the available JET EDGE LIDAR system is applicable for in situ analysis of deposited layers of the JET divertor.

Further development of the available EGDE LIDAR Laser System and optimisation of LIBS analytical features may result in a fruitful realisation of a permanent LIBS system.

\section{Acknowledgements}

The authors are grateful to the JET administration for authorization to perform a set of LIBS studies on JET installation with EGDE LIDAR Laser System.

\section{References}

[1] G. Counsell, P. Coad, C. Grisolia, et al., Tritium retention in next step devices and the requirements for mitigation and removal techniques, Plasma Phys. Controlled Fusion 48 (2006) B189-B199.

[2] C. Grisolia, S. Rosanvallon, P. Coad, et al., JET contributions to ITER technology issues, Fusion Eng. Des. 81 (2006) 149-154.

[3] C. Grisolia, G. Counsell, G. Dinescu, et al., Treatment of ITER plasma facing components: current status and remaining open issues before ITER implementation, Fusion Eng. Des. 82 (2007) 2390-2398.

[4] T. Loarer, Fuel retention in tokamaks, J. Nucl. Mater. 390-391 (2009) 20-28.

[5] F. Le Guern, F. Brygo, P. Fichet, et al., Co-deposited layer characterisation and removal control by optical emission spectroscopy coupled to nanosecond laser ablation, Fusion Eng. Des. 81 (2006) 1503-1509.

[6] A. Semerok, S.V. Fomichev, J.M. Weulersse, et al., Heating and ablation of tokamak graphite by pulsed nanosecond Nd-YAG lasers, J. Appl. Phys. 101 (2007) 084916.

[7] L. Mercadier, J. Hermann, C. Grisolia, et al., Plume segregation observed in hydrogen and deuterium containing plasmas produced by laser ablation of carbon fiber tiles from a fusion reactor, Spectrochim, Acta Part B 65 (2010) 715-720.

[8] L. Mercadier, A. Semerok, P.A. Kizub, et al., In-depth analysis of ITER-like samples composition using laser-induced breakdown spectroscopy, J. Nucl. Mater. 414 (2011) 485-491.

[9] L. Mercadier, J. Hermann, C. Grisolia, et al., Analysis of deposited layers on plasma facing components by laser-induced breakdown spectroscopy: towards ITER tritium inventory diagnostics, J. Nucl. Mater. 415 (2011) S1187.

[10] A. Semerok, S.V. Fomichev, F. Brygo, et al., Pulsed repetition rate nanosecond laser heating and ablation of the tokamak graphite tile deposited layers, J. Nucl. Mater. 420 (2012) 198-206.

[11] L. Mercadier, J. Hermann, C. Grisolia, et al., Diagnostics of nonuniform plasmas for elemental analysis via laser-induced breakdown spectroscopy: demonstration on carbon-based materials, J. Anal. At. Spectrom. 28 (2013) 1446-1455.

[12] A. Semerok, C. Grisolia, LIBS for tokamak plasma facing components characterisation: perspectives on in situ tritium cartography, Nucl. Instrum. Methods Phys. Res., Sect. A 720 (2013) 31-35.

[13] S. Almaviva, L. Caneve, F. Colao, et al., Remote-LIBS characterization of ITER-like plasma facing materials, J. Nucl. Mater. 421 (2012) 73-79.

[14] S. Almaviva, L. Caneve, F. Colao, et al., Laboratory feasibility study of fusion vesse inner wall chemical analysis by laser induced breakdown spectroscopy, Chem. Phys. 398 (2012) 228-232.

[15] S. Almaviva, L. Caneve, F. Colao, et al., Double pulse laser induced breakdown spectroscopy measurements on ITER-like samples, Fusion Eng. Des. 96-97 (2015) 848-851.
[16] S. Almaviva, L. Caneve, F. Colao, et al., Measurements of deuterium retention and surface elemental composition with double pulse laser induced breakdown spectroscopy, Phys. Scr. T167 (2016) 014043.

[17] V.M. Kozhevin, E.E. Mukhin, G.T. Razdobarin, et al., Laser induced breakdown spectroscopy technique for in-situ dust detecting in a next-step tokamak, in "Hydrogen and Helium Recycling at Plasma Facing Materials", NATO Science Series II: Mathematics, Physics and Chemistry, vol. 54, pp. 213-226, published 2002, Edited by A. Hassanein.

[18] E. Mukhin, K. Vukolov, V. Semenov, et al., Progress in the development of deposition prevention and cleaning techniques of in-vessel optics in ITER, Nucl. Fusion 49 (2009) 085032.

[19] P. Gasior, M. Bieda, M. Kubkowska, et al., Laser induced breakdown spectroscopy as diagnostics for fuel retention and removal and wall composition in fusion reactors with mixed-material components, Fusion Eng. Des. 86 (2011) 1239-1242.

[20] G. Shaw, M.Z. Martin, R. Martin, et al., Preliminary design of laser-induced breakdown spectroscopy for proto-material plasma exposure eXperiment, Rev. Sci. Instrum. 85 (2014) 11D806.

[21] C. Li, D. Zhao, X. Wu, H. Ding, Spatial resolution measurements of C, Si and Mo using LIBS for diagnostics of plasma facing materials in a fusion device, Plasma Sci. Technol. 17 (2015) 638-643.

[22] C. Li, D. Zhao, Z. Hu, X. Wu, G.-N. Luo, J. Hu, H. Ding Characterization of deuterium retention and co-deposition of fuel with lithium on the divertor tile of EAST using laser induced breakdown spectroscopy, J. Nucl. Mater. 463 (2015) 915-918.

[23] H.J. van der Meiden, M.A. van den Berg, S. Brons, et al., Laser-based diagnostics applications for plasma-surface interaction studies, J. Instrum. 8 (2013) C11011.

[24] A. Malaquias, V. Philipps, A. Huber, et al., Development of ITER relevant laser techniques for deposited layer characterisation and tritium inventory, J. Nucl. Mater. 438 (2013) S936-S939.

[25] Q. Xiaoa, A. Huber, G. Sergienko, et al., Application of laser-induced breakdown spectroscopy for characterization of material deposits and tritium retention in fusion devices, Fusion Eng. Des. 88 (2013) 1813-1817.

[26] A. Huber, B. Schweer, V. Philipps, et al., Development of laser-based diagnostics for surface characterisation of wall components in fusion devices, Fusion Eng. Des. 86 (2011) 1336-1340.

[27] P. Paris, K. Piip, A. Hakola, et al., Development of laser induced breakdown spectroscopy for studying erosion, deposition, and fuel retention in ASDEX upgrade, Fusion Eng. Des. 98-99 (2015) 1349-1352.

[28] J. Karhunen, A. Hakola, J. Likonen, et al., Applicability of LIBS for in situ monitoring of deposition and retention on the ITER-like wall of JET - comparison to SIMS, J. Nucl. Mater. 463 (2015) 931-935.

[29] B. Sallé, J.-L. Lacour, E. Vors, et al., Laser-induced breakdown spectroscopy for Mars surface analysis: capabilities at stand-off distances and detection of chlorine and sulfur elements, Spectrochim. Acta Part B 59 (2004) 1413-1422.

[30] B. Sallé, T. David, D.A. Cremers, et al., Laser-induced breakdown spectroscopy for space exploration applications: influence of the ambient pressure on the calibration curves prepared from soil and clay samples, Spectrochim. Acta Part B 60 (2005) 479-490.

[31] B. Sallé, J.-L. Lacour, P. Mauchien, et al., Comparative study of different methodologies for quantitative rock analysis by laser-induced breakdown spectroscopy in a simulated Martian atmosphere, Spectrochim. Acta Part B 61 (2006) 301-313.

[32] M. Kempenaars, J.C. Flanagan, L. Giudicotti, et al., Enhancement of the JET edge LIDAR Thomson scattering diagnostic with ultrafast detectors, Rev. Sci. Instrum. 79 (2008) $10 \mathrm{E} 728$.

[33] NIST database, http://www.nist.gov.

[34] A. Semerok, P. Mauchien, Ultrashort pulse laser ablation for surface elemental analysis, Rev. Laser Eng. 33 (2005) 5308-5336.

[35] A. Semerok, C. Dutouquet, Ultrashort double pulse laser ablation of metals, Thin Solid Films 453-454 (2004) 501-505.

[36] A. Semerok, C. Dutouquet, Analytical performances of laser-induced micro-plasma of Al samples with single and double ultrashort pulses in air and with Ar-jet: a comparative study, Spectrochim. Acta Part B 99 (2014) 163-171.

[37] M. Fabert, L. Gallais, Y. Pontillon, On-line deformation measurements of nuclear fuel rod cladding using speckle interferometry, Prog. Nucl. Energy 72 (2014) 44-48.

[38] E. Tognoni, G. Cristoforetti, S. Legnaioli, et al., Calibration-free laser-induced breakdown spectroscopy: state of the art, Spectrochim. Acta Part B 65 (2010) 1-14.

[39] J. Hermann, A. Lorusso, A. Perrone, et al., Simulation of emission spectra from nonuniform reactive laser-induced plasmas, Phys. Rev. E92 (2015) 053103.

[40] T. Tanabe, N. Bekris, P. Coad, et al., Tritium retention of plasma facing components in tokamaks, J. Nucl. Mater. 313-316 (2003) 478-490.

[41] N. Bekris, J.P. Coad, C. Grisolia, et al., Fusion technology related studies at JET: postmortem tile analysis with MKII-HD geometry, in situ laser detritiation and molecular sieve bed detritiation, J. Nucl. Mater. 417 (2011) 1356-1360.

[42] J.D. Hey, C.C. Chu, J.P.S. Rash, Partial local thermal equilibrium in a low-temperature hydrogen plasma, J. Quant. Spectrosc. Radiat. Transf. 62 (1999) 371-387.

[43] J.C.S. Kools, T.S. Baller, S.T. De Zwart, J. Dieleman, Gas flow dynamics in laser ablation deposition, J. Appl. Phys. 71 (1992) 4547-4556.

[44] S.I. Anisimov, B.S. Luk'yanchuk, A. Luches, An analytical model for three-dimensional laser plume expansion into vacuum in hydrodynamic regime, Appl. Surf. Sci. 96-98 (1996) 24-32.

[45] W. Pietsch, A. Petit, A. Briand, Isotope ratio determination of uranium by optical emission spectroscopy on a laser-produced plasma - basic investigations and analytical results, Spectrochim. Acta Part B 53 (1998) 751-761.

[46] X. Ni, K.K. Anoop, X. Wang, et al., Dynamics of femtosecond laser-produced plasma ions, Appl. Phys. A Mater. Sci. Process. 117 (2014) 111-115. 
[47] A.A. Morozov, Analytical formula for interpretation of time-of-flight distributions for neutral particles under pulsed laser evaporation in vacuum, J. Phys. D. Appl. Phys. 48 (2015) 19550.

[48] C.G. Parigger, E. Oks, Hydrogen Balmer series spectroscopy in laser-induced breakdown plasmas, Int. Rev. At. Mol. Phys. 1 (2010) 13-23.

[49] A.N. Zaidel', V.K. Prokof'ev, S.M. Raiskii, V.A. Slavnyi, E.Y. Shreider, Table of Spectral Lines, Springer Science + Business Media, New York, 1970

[50] J.D. Hey, C.C. Chu, E. Hintz, On a heating mechanism for cold hydrogen and deuterium atoms produced at the edge of a tokamak plasma, J. Phys. B Atomic Mol. Phys. 32 (1999) 3555-3573.

[51] J.D. Hey, M. Korten, Y.T. Lie, et al., Doppler broadening and magnetic field effects on the Balmer lines emitted at the edge of a tokamak plasma, Contrib. Plasma Phys. 36 (1996) 583-604.

[52] J. Rosato, V. Kotov, D. Reiter, Modelling of passive spectroscopy in the ITER divertor: the first hydrogen Balmer lines, J. Phys. B Atomic Mol. Phys. 43 (2010) 144024.
[53] V.N. Rai, A.K. Rai, F.-Y. Yueh, et al., Optical emission from laser-induced breakdown plasma of solid and liquid samples in the presence of a magnetic field, Appl. Opt. 42 (2003) 2085-2093.

[54] R. Hai, P. Liu, D. Wu, Q. Xiao, L. Sun, H. Ding, Effect of steady magnetic field on laserinduced breakdown spectroscopic characterization of EAST-like wall materials, J. Nucl. Mater. 463 (2015) 927-930.

[55] P. Liu, R. Hai, D. Wu, et al., The enhanced effect of optical emission from laser induced breakdown spectroscopy of an Al-Li alloy in the presence of magnetic field confinement, Plasma Sci. Technol. 17 (2015) 687-692.

[56] Q. Xiao, R. Hai, H. Ding, et al., In-situ analysis of the first wall by laser-induced breakdown spectroscopy in the TEXTOR tokamak: dependence on the magnetic field strength, J. Nucl. Mater. 463 (2015) 911-914.

[57] A. Semerok, A. Pailloux, CW laser-induced fluorescent spectroscopy of highly magnetized collisionless plasma, Laser Phys. 9 (1999) 244-248.

[58] A. Semerok, B. Larousse, A. Pailloux, Optical diagnostics system for measuring SUPER-ERIC plasma parameters, Laser Phys. 10 (2000) 372-377. 